\title{
Death associated protein 1 is correlated with the clinical outcome of patients with colorectal cancer and has a role in the regulation of cell death
}

\author{
YONGNING JIA ${ }^{1-3}$, LIN YE ${ }^{1,2}$, KE JI ${ }^{1,2}$, ANN-MARIE TOMS ${ }^{1,2}$, MANSEL LEIGH DAVIES ${ }^{1,2}$, \\ FIONA RUGE $^{1,2}$, JIAFU JI ${ }^{1,3}$, RACHEL HARGEST ${ }^{1,2}$ and WEN G. JIANG ${ }^{1,2}$ \\ ${ }^{1}$ Cardiff University-Peking University School of Oncology Joint Institute; ${ }^{2}$ Metastasis and Angiogenesis Research Group, \\ Institute of Cancer and Genetics, Cardiff University School of Medicine, Heath Park, Cardiff CF14 4XN, UK; \\ ${ }^{3}$ Key Laboratory of Carcinogenesis and Translational Research (Ministry of Education), Department of \\ Gastrointestinal Surgery, Peking University Cancer Hospital and Institute, Haidian, Beijing 100142, P.R. China
}

Received October 14, 2013; Accepted November 1, 2013

DOI: $10.3892 /$ or.2013.2866

\begin{abstract}
Death-associated protein 1 (DAP1) is a member of the DAP family and has been implicated in the regulation of cell growth and death including that of cancer cells. However, the roles of DAP1 in clinical cancer and in the regulation of colorectal cancer cells are largely unknown. The present study investigated the expression profile of DAP1 in human colorectal cancer and the impact of DAP1 on apoptosis and the cellular response to 5-FU. Human colorectal cancer specimens $(\mathrm{n}=94)$ and human colorectal cancer cell lines HRT18 and HT115 were used. DAP1 transcript and protein were evaluated using quantitative transcript analysis and immunohistochemistry. DAP1-knockdown cells were generated using anti-DAP1 transgene. The results revealed that human colorectal cancer tissues had lower levels of DAP1 when compared with the normal tissues. The reduced levels were associated with higher Dukes' stage and lymph node metastasis. Patients with low DAP1 expression had a markedly reduced survival. Loss of DAP1 in colorectal cancer cells resulted in a gain in cellular migration and loss of their sensitivity of apoptosis to chemotherapeutic agent, 5-FU. DAP1 was found to be correlated with disease progression and long-term survival of the colorectal patients. DAP1 is also a pivotal regulator of the growth and apoptosis and cellular response to chemotherapy agents.
\end{abstract}

\section{Introduction}

DAP1 is one of the members of the death-associated protein (DAP) family, first isolated as a gene involved in IFN- $\gamma$-induced apoptosis through a technical knockout strategy (TKO), i.e.

Correspondence to: Professor Wen G. Jiang, Metastasis and Angiogenesis Research Group, Institute of Cancer and Genetics, Cardiff University School of Medicine, Heath Park, Cardiff CF14 4XN, UK E-mail: jiangw@cf.ac.uk

Key words: death associated protein 1, colorectal cancer, Dukes' staging, survival, cell growth, apoptosis random inactivation of genes with antisense cDNA libraries (1). The DAP family is comprised of DAP1, DAP2 (DAP kinase), DAP3, DAP4 and DAP5. DAP2 was found to be involved in apoptosis induced by IFN- $\gamma$, TNF- $\alpha$ and Fas (2-3). Serine/threonine kinase catalytic activity and the death domain contribute to the pro-apoptotic function. Based on the loss of DAP2 expression in certain types of cancers, it has been proposed as a candidate tumour-suppressor gene. While the clinical significance of other members of the DAP Family that also exhibit death-promoting functions remain largely unknown, there have been recent reports on the possible association between the DAP family and the clinical outcome of patients with malignant diseases, for example breast cancer (4).

Induction of apoptosis is an important mechanism of chemotherapeutic agents in the treatment of cancer. Among the DAP family members, DAP3 was found to be a mitochondrial protein that has a specific amino acid sequence to recognise and anchor to mitochondria. Mitochondria play a key role in apoptosis including release of pro-apoptotic substances such as cytochrome $c$ and AIF, production of reactive oxygen species (ROS) and reduction in ATP synthesis (5-8). All of these findings indicate that DAP may be involved in both the intrinsic and extrinsic apoptosis pathways. In addition, it was reported that DAP2 methylation is associated with a reduced response to chemotherapy and poor prognosis in gastric cancer (9).

In the present study, we investigated the expression pattern of DAP1 in a cohort of colorectal cancer patients. Decreased expression of DAP1 transcripts was detected in tumour tissues. qPCR analysis revealed that DAP1 expression levels were correlated with clinical and pathological parameters of the colorectal cancer patients including disease-free survival. Furthermore, the study reports the role of DAP1 in the growth and apoptosis of colorectal cancer cells and in the cellular response of cells to chemotherapeutic agents.

\section{Materials and methods}

Materials. HRT18 and HT115 cell lines were obtained from the European Collection of Cell Cultures (ECACC; Salisbury,UK). 
Reagents and kits were obtained from Promega Corporation (Madison, WI, USA), Bio-Rad Lifescience Company (Hercules, CA, USA), and Gibco Invitrogen Corporation (Gibco-BRL, Paisley, Scotland, UK). The secondary and goat polyclonal antibodies raised against a peptide mapping at the N-terminus of human DAP1 were purchased from Santa Cruz Biotechnology, Inc. (Santa Cruz, CA, USA).

Colorectal cancer tissues were collected immediately after surgery. All protocols were reviewed and approved by the local ethics committee, and consent was obtained from the patients.

Tissue processing, RNA extraction, cDNA synthesis and $R T-P C R$. Frozen sections of the tissues were cut to thicknesses of 5-10 $\mu \mathrm{m}$ and kept for immunohistochemistry and routine histology (10). RNA was isolated using Total RNA reagent (Promega Corporation). cDNA synthesis and RT-PCR were performed using standard methods.

Quantitative analysis of DAP1. DAP1 mRNA expression in the colon cancer tissues was determined by quantitative realtime PCR, based on the Amplifluor ${ }^{\mathrm{TM}}$ technology as previously described (11). DAP1 qPCR primers as follows were designed using Beacon Designer software (Premier Biosoft, Palo Alto, CA, USA): sense, 5'-ATGGACAAGCATCCCTTCC-3' and antisense, 5'-ACTGAACCTGACCGTACACTCTGTCAGG GAAATACCAA-3', exon 10-12). The underlined sequence is complementary to the universal $\mathrm{Z}$ probe (TCS Biologicals Ltd., Oxford, UK).

Immunohistochemical staining of the DAP1 protein. Frozen sections of tissues were cut to thicknesses of 5-10 $\mu \mathrm{m}$, and IHC was performed using the anti-DAP1 antibody and Vectastain ABC kits (Vector Laboratories, Burlingame, CA, USA).

Ribozyme transgene targeting human DAP1. Anti-human DAP1 hammerhead ribozymes were designed according to the secondary structure of the gene (Fig. 2A) using the Zuker RNA mFold program (sense, 5'-CTGCAGTTCAACCACTT CTCCCAGAGCTGATGAGTCCGTGAGGA-3' and antisense, 5'-ACTAGTAGAGAAAGCACTGAGAAAGGGAGT TTCGTCCTCACGGACT-3') (12). The ribozymes were accordingly synthesised using touchdown PCR and cloned into the pEF6/V5-His TOPO TA Expression kit (Invitrogen) following the manufacturer's protocol. Transfection was performed using an Easyject Plus electroporator (EquiBio, Kent, UK). After up to 5 days of selection with blasticidin, the transfectants from the DAP1 knockdown and control cells were verified using RT-PCR and then used in the subsequent experiments.

Western blot analysis. The protein concentrations in the cell lysates were determined using the DC protein assay kit (Bio-Rad) and an ELx800 spectrophotometer (BioTek). Proteins were probed with the anti-DAP1 (1:200) and anti-GAPDH-antibodies (1:500) (both from Santa Cruz Biotechnology, Inc.) as an internal control, followed by a peroxidase-conjugated secondary antibody $(1: 1,000)$. Protein bands were visualised and photographed using an UVITech imager (UVITech, Inc.).
In vitro cell growth assay under normal culture condition, or following exposure to gradient concentrations of $5 F U$ and oxaliplatin. Equal amounts of cells were plated into 96-well plates at 2,500 cells/well with a gradient concentration of a combination of 5FU and oxaliplatin at a 1:5 dilution. The dilution started from 10 times that of the threshold concentration $\left(2 \times 10^{-6}\right)$. Cell growth was assessed after 1, 3 and 5 days. Crystal violet was used to stain the cells, and the absorbance was determined at a wavelength of $540 \mathrm{~nm}$ using a spectrophotometer ELx800.

The blood and tissue fluid drug concentrations of $5 \mathrm{FU}$ and oxaplatin were determined to be $5 \times 10^{-5} \mathrm{M}$ and $1 \times 10^{-4} \mathrm{M}$, respectively, according to the drug dosage used in clinical chemotherapy regimens. The wild-type HRT18 and HT115 cells were plated with a gradient concentration with a 1:5 dilution of 5 times that of the blood concentration. The concentration causing obviously increased cell growth was set as a threshold dosage.

Cell matrix adhesion assay. The cell matrix adhesion assay was conducted as previously described (13). Cells were added to a 96-well plate precoated with Matrigel (Collaborative Research Products, Bedford, MA, USA) (5 $\mu \mathrm{g} /$ well). After $40 \mathrm{~min}$ of incubation, the non-adherent cells were washed off using BSS buffer. The remaining cells were fixed, stained and counted.

Wound healing assay. The wound healing assay was performed as previously described (14). The monolayer of cells was scraped with a fine gauge needle. The movement of cells to close the wound was recorded on a time lapse video recorder and analysed using Optimas 6.0 motion analysis (Meyer Instruments, Inc., Houston, TX, USA).

In vitro invasion assay. The in vitro invasion assay was performed as previously described (14). Transwell inserts with an $8-\mu \mathrm{m}$ pore size were coated with $50 \mu \mathrm{g}$ of Matrigel and air-dried. Following rehydration, 40,000 cells were added to each well. After 3 days of incubation, cells that had migrated through the matrix to the other side of the insert were fixed, stained and counted.

Flow cytometric analysis of apoptosis following treatment with a combination of 5FU and oxaliplatin. DAP1-RIB and empty vector PEF-transfected HRT18 and HT115 cells were plated into a 6 -well plate at a density of $3 \times 10^{5}$ cells/well. Each cell line was plated into 3-wells at the same time. The first well was used as the control with no treatment, and the second and third wells received treatment with a combination of 5FU and oxaliplatin at concentrations of $2 \times 10^{-6} \mathrm{M}$ and $1 \times 10^{-5} \mathrm{M}$, respectively. All cells, including those floating in the culture medium were harvested after $6 \mathrm{~h}$ of incubation. The apoptotic population was determined using a Vybrant ${ }^{\circledR}$ Apoptosis Assay kit and flow cytometry and FlowMax software package as previously described (15).

Statistical analysis. Statistical analysis was performed using SPSS software (SPSS standard version 13.0; SPSS Inc.). The relationship between DAP1 expression and tumor grade, TNM stage and nodal status were assessed using Mann-Whitney 
U test and Kruskal-Wallis test. (The error bar shown in the graph represents SEM). Survival curves were analyzed using Kaplan-Meier survival analysis. Differences were considered statistically significant at $\mathrm{p}<0.05$.

\section{Results}

DAPI mRNA expression in the colorectal adenocarcinoma tissues. DAP1 transcript expression was examined in specimens from the 94 colorectal adenocarcinoma patients using real-time quantitative PCR (expressed as mean DAP1 transcript copies/ $\mu 1$ of RNA from $50 \mathrm{ng}$ total RNA and standardized with GAPDH). The cohort comprised 61 men (71.8\%) and 24 women (28.2\%). The average age of all patients was 56.9 years. A significantly lower mRNA expression level of DAP1 was observed in the tumour tissues when compared with the normal background tissues $(\mathrm{p}=0.027)$.

DAP1 expression correlates with tumour invasiveness and clinical stage. The relationship between DAP1 expression and pathological status was also assessed in the present study through quantitative analysis of DAP1 transcript. DAP1 levels were initially assessed in relation to tumour invasiveness. Invasive tumours appeared to have reduced levels of DAP1 when compared with non-invasive tumours, but the difference did not reach statistical significance.

The relationship between DAP1 expression and clinical TNM stage is shown in Table I. The DAP1 expression level was relatively higher in lymph node-negative patients in comparison with the lymph node-positive group. Although the difference was not significant $(\mathrm{p}=0.21)$, a trend was observed that DAP1 expression decreased along with the upgrading of $\mathrm{T}$ stage $(\mathrm{p}=0.218)$. Expression levels of DAP1 were also decreased in the advanced disease cases. Statistical analysis revealed a significant difference $(p=0.039)$. This is in line with the reduced expression of DAP1 in tumours of Dukes' $\mathrm{B}$ and $\mathrm{C}$ groups, which was significantly lower than its level in tumours of the Dukes' A group $(\mathrm{p}=0.036)$.

DAPl expression in relation to recurrence and metastasis. The correlations between DAP1 expression levels and occurrence of incidents (local recurrence and distant metastasis) were also investigated. We found a significant correlation between DAP1 expression levels and the incidents $(\mathrm{p}=0.037)$ (Table I).

Quantitative studies showed that DAP1 expression levels in patients with either recurrence or metastasis were significantly lower than those without any of these complications.

Correlation between DAPI expression and survival of the colorectal adenocarcinoma patients. The median follow-up period was 21.7 months (0.7-88 months) for the current cohort. According to a threshold level (average DAP1 level of Dukes' B), Kaplan-Meier analysis demonstrated that patients with higher levels of DAP1 expression in tumours had a prolonged overall survival (Fig. 1A) and longer disease-free survival time (Fig. 1B) when compared with the lower expression level group $(\mathrm{p}<0.01)$.

In the stratified survival analysis according to node status, node-negative patients with high DAP1 levels had a significantly prolonged survival in comparison with the low level
Table I. DAP1 transcript levels in colorectal tumour tissues.

DAP1 transcripts

(copies/50 ng RNA) P-value

means $\pm \mathrm{SD}$

$\begin{array}{lr}\text { Location } & \\ \text { Left colon } & 1,803 \pm 2,749 \\ \text { Right colon } & 845 \pm 2,046 \\ \text { Transcolon } & 0.426 \pm 0.531 \\ \text { Rectum } & 82 \pm 1,063 \\ \text { Differentiation } & \\ \text { Well-differentiated } & 5,099 \pm 7,207 \\ \text { Moderately differentiated } & 709 \pm 1,614 \\ \text { Poorly differentiated } & 1,859 \pm 2,836 \\ \text { Dukes' stage } & \\ \text { A } & 3,203 \pm 3,975 \\ \text { B } & 995 \pm 2,192 \\ \text { C } & 689 \pm 1,602 \\ \text { T stage } & \\ \text { T1 } & 3,589 \pm 2,404 \\ \text { T2 } & 1,944 \pm 3,587 \\ \text { T3 } & 872 \pm 2,008 \\ \text { T4 } & 801 \pm 1,764\end{array}$

0.744

0.007

0.036

$\mathrm{N}$ stage

Negative

Positive

$1,379 \pm 1,443$

$711 \pm 1,623$

TNM stage

I

II

$3,071 \pm 3,646$

$913 \pm 2,142$

$537 \pm 1,532$

III

$1,347 \pm 1,877$

Invasiveness

Non-invasive

$72.1 \pm 188.7$

Invasive

$46.7 \pm 110.4$

Incidents

Disease-free

$1,441 \pm 2,695$

Incidents

$335 \pm 1,013$

(recurrence and metastases)

Survival

0.14

Alive

$1,384 \pm 2,672$

$570 \pm 1,404$

Death

Distant metastasis

$586 \pm 1,495$

$1,229 \pm 2,398$

No

0.016

Recurrence

$1,111 \pm 2,307$

No recurrence

$206 \pm 495$

0.039

$\mathbf{0 . 0 3 7}$

Local recurrence

DAP1 transcript levels were normalized against the corresponding CK19 expression. Values are shown as means \pm standard deviation (SD) DAP1, death-associated protein 1 . Bold text, statistically significant. 
A

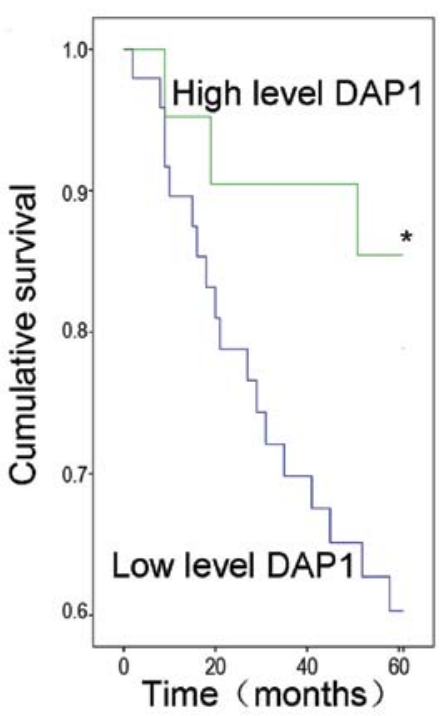

B

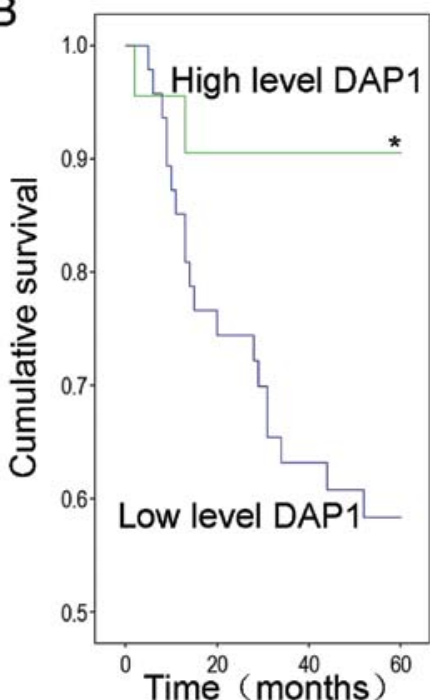

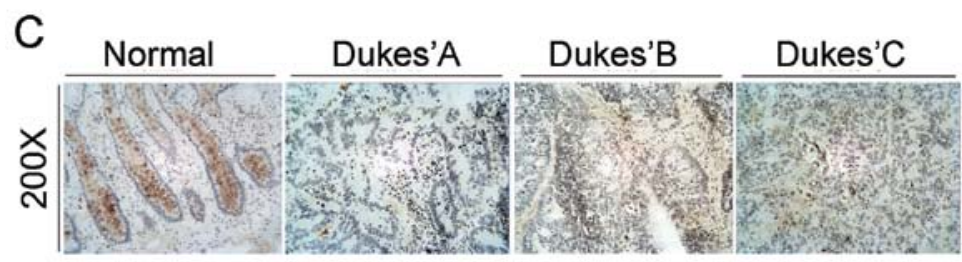

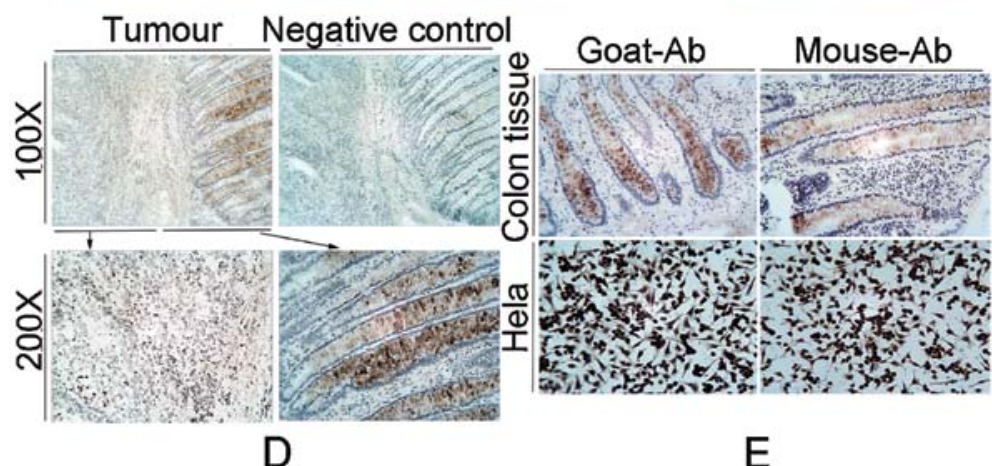

Figure 1. DAP1 expression and correlation with prognosis. (A) Correlation of DAP1 expression with overall survival. (B) Correlation of DAP1 expression with disease-free survival. " $\mathrm{p}<0.01$. (C) Immunohistochemical staining of DAP1 in normal colon tissues, and tumours of Dukes' stage A, B and C. (D) DAP1 staining in colon specimens showing strong staining in the adjacent background normal epithelial cells which was weak or absent in the cancer cells. (E) Results of goat anti-DAP1 and mouse anti-DAP1 antibodies in colon tissues and HeLa cells. Images were reduced from images captured under a lens at x200. Negative control was performed using the secondary antibody alone. DAP1, death-associated protein 1.

group ( $\mathrm{p}=0.011)$. In the node-positive patients, no significant association was found between DAP1 expression and survival. Finally, multivariate analysis using gender, age, grade, TNM, nodal status and DAP1 expression levels as variants showed that nodal status $(\mathrm{p}=0.015), \mathrm{TNM}(\mathrm{p}=0.006)$, grade $(\mathrm{p}=0.026)$, age $(\mathrm{p}=0.020)$ and DAP1 $(\mathrm{p}=0.037)$ are all independent factors for overall survival.

Immunohistochemical staining of human colorectal adenocarcinoma specimens. To assess the expression pattern of DAP1 at the protein level, we performed immunohistochemical analysis of DAP1 in the human colorectal adenocarcinoma tissue sections ( $n=20$ pairs). Using a specific anti-DAP1 polyclonal antibody, DAP1 was detected both in the cytoplasm of the tumours and in the non-tumour cells. The staining of DAP1 was significantly reduced or absent in the tumour tissues when compared with the non-tumour tissues. No obvious staining of DAP1 was observed in the stromal cells in either normal or tumour tissues (Fig. 1C-E).

Stable knockdown of DAP1. To investigate the role of DAP1 in colorectal adenocarcinoma, knockdown of DAP1 expression was performed in the HRT18 and HT115 cell lines which expressed DAP1. Reduced transcript levels of DAP1 were verified in the HRT18 and HT115 cells which were transfected with ribozyme transgenes using RT-PCR (Fig. 2B). A reduction in the protein expression of DAP1 was further confirmed using western blotting (Fig. 2C). These DAP1-modified cell lines were used for the following in vitro studies.

Impact of DAP1 knockdown on in vitro cell growth following treatment with gradient concentrations of $5 F U$ and oxaliplatin. Although there was no significant difference noted in the DAP1-knockdown cells in comparison with the 

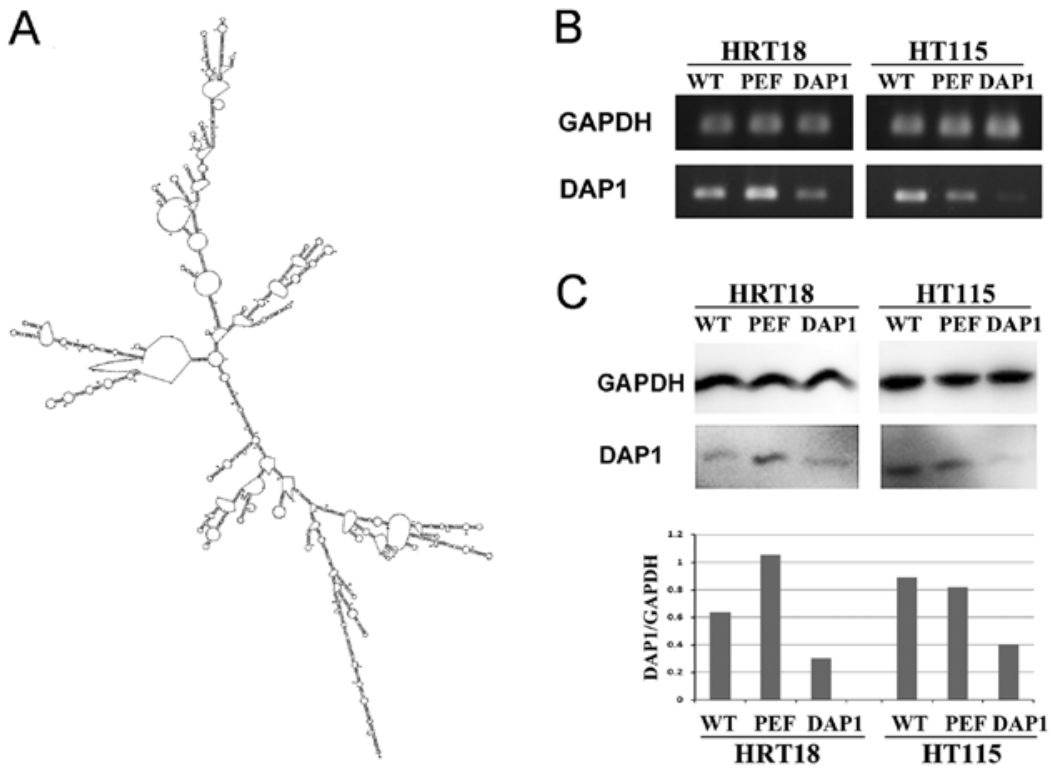

Figure 2. Confirmation of DAP1 expression knockdown in HRT18 and HT115 cell lines. (A) Secondary structure of DAP1 mRNA. (B) Expression of DAP1 at the mRNA level in the 2 cell lines. (C) Expression of DAP1 at the protein level in the 2 cell lines. The protein band volume (integrated density) was quantified using ImageJ. Bar graph shows the ratio of DAP1 to GAPDH of each cell line. DAP1, death-associated protein 1.

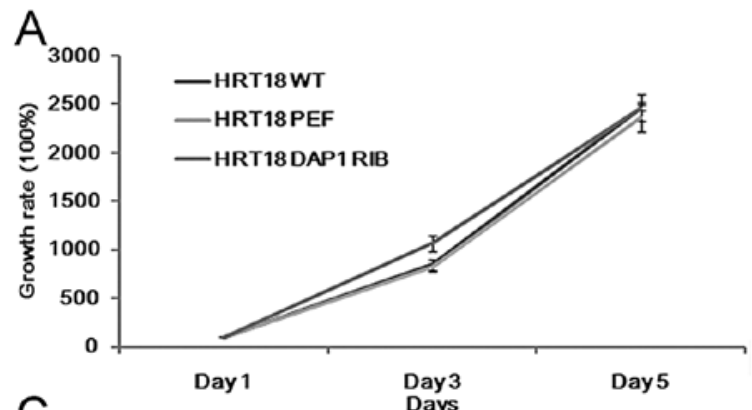

C

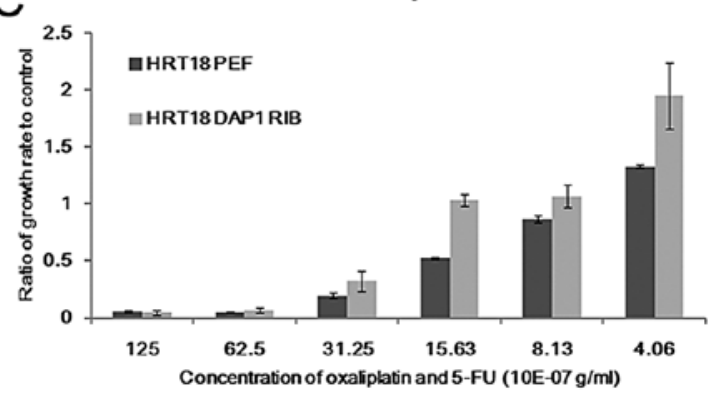

$\mathrm{B}$
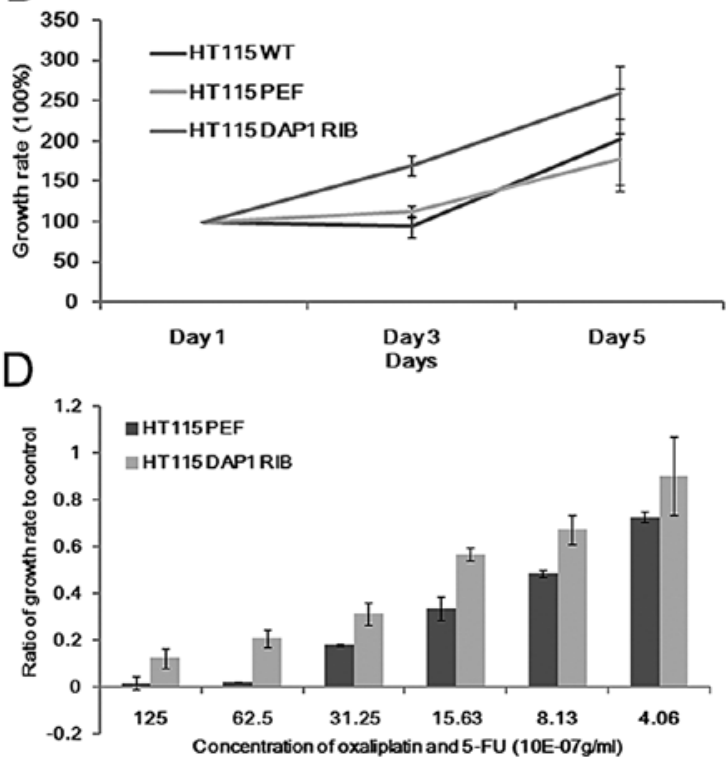

Figure 3. In vitro growth and drug sensitivity tests of the DAP1-RIB cell lines and the corresponding control cell lines. (A and B) In vitro growth assay of the HRT18 and HT115 cell lines. (C and D) In vitro drug sensitivity assay of the HRT18 and HT115 cell lines. DAP1, death-associated protein 1.

controls in regards to cell growth (Fig. 3A and B), these cells appeared to be less responsive to treatment with $5 \mathrm{FU}$ alone or in combination with oxaliplatin. The growth rate of DAP1-RIB cells significantly exceeded that of the control cells. This effect was noted in both the HRT18 and HT115 cell lines (Fig. 3C and D).

Effects of DAPI knockdown on adhesion, migration and invasion of colorectal cancer cells. We further examined the influence of DAP1 on the adhesive nature of the colorectal cancer cells (Fig. 4A and B). Knockdown of DAP1 expression resulted in an increase in adhesive ability of both HRT18 and HT115 cells.

A wound healing assay was employed to examine the influence of DAP1 knockdown on migration. Downregulation of DAP1 significantly promoted cell migration to close the wound when compared with the control in the HRT18 cell line $(\mathrm{p}<0.05)$. The migration was also promoted by DAP1 knockdown to a lesser degree in the HT115 cell line (Fig. 4C and D).

Finally, the presence of DAP1 has also been shown to affect colorectal cancer cell invasion. Knockdown of DAP1 in the HT115 cell line resulted in a marked increase in cell inva- 

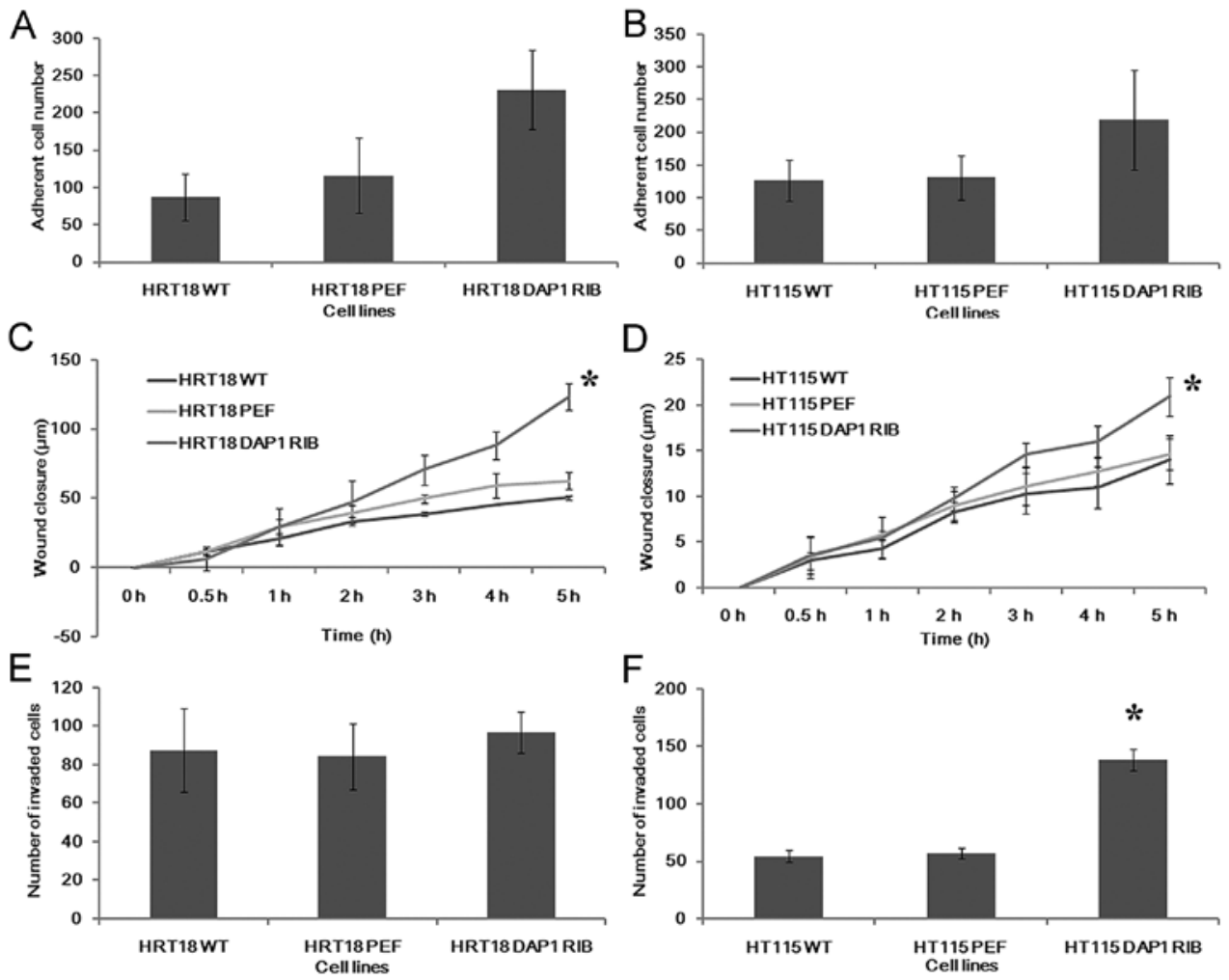

Figure 4. In vitro adhesion, migration and invasion cell function assays of the DAP1-RIB cell lines and corresponding control cell lines. (A and B) In vitro adhesion assay in HRT18 and HT115 cell lines. (C and D) In vitro wound healing assay in the HRT18 and HT115 cell lines. (E and F) In vitro invasion assay in the HRT18 and HT115 cell lines. ${ }^{*}$ p $<0.01$. DAP1, death-associated protein 1.

siveness ( $\mathrm{p}<0.0001$ vs. controls). However, no similar effect was observed in the HRT18 cell line (Fig. 4E and F).

Effects of DAPI knockdown on apoptosis in response to chemotherapeutic agents. As shown in Fig. 5, when compared to the control group, the percentage of cells undergoing apoptosis was significantly higher in both the HRT18 and HT115 cells treated with the chemotherapeutic agents. The apoptotic population was increased in a concentration-dependent manner. In comparison with the cells transfected with empty vectors, both HRT18DAP1RIB and HT115DAP1RIB cells consisted of a significantly lower percentage of apoptotic cells when exposed to different concentrations of the chemotherapeutic agents (Fig. 5).

\section{Discussion}

DAP1 belongs to the DAP family which was initially isolated through technical knockout (TKO) strategy, and was characterized as an apoptosis-associated protein because of its involvement in IFN- $\gamma$-induced apoptosis. The DAP family consists of DAP1, DAP2 (DAP kinase), DAP3, DAP4 and DAP5. These members share some common feature domains which confer a pro-apoptotic function. However, the expression pattern of the DAP family differs among different types of human tumours. DAP2 has been reported to be below the limit of detection in $80 \%$ of B cell leukemia cell lines, while the frequency of loss of DAP2 expression in breast, bladder and renal carcinoma cell lines ranges from 30 to $40 \%$ (16) Promoter hypermethylation of DAP2 was also reported in various types of human tumours, including B cell lymphoma, non-small cell lung, head and neck and colon cancer $(17,18)$. DAP3 was found to be overexpressed in human thyroid oncocytic tumours and correlated with human thymoma stage $(19,20)$. In breast cancer, however, DAP3 was found to be lost in aggressive tumours, and low levels of DAP-3 were found to be linked to a shorter survival of breast cancer patients (20). In the present study, the DAP1 expression pattern in colorectal adenocarcinoma was similar to that of DAP2. Immunohistochemistry revealed that DAP1 expression in tumour cells was significantly lower than that of the background normal tissues. qPCR analysis of DAP1 in our clinical colorectal cancer cohort revealed that lower expression of DAP1 was correlated with lymph node involvement, higher $\mathrm{T}$ stage, tumour invasiveness, local recurrence, metastases, higher TNM stage and higher Dukes' stage. A higher level of DAP1 expression indicated a longer disease-free survival time. From the above data and reports of similar research, it can be argued that DAP1 acts as a tumour-suppressor gene in colorectal cancer.

In order to clarify the role of DAP1 in colorectal cancer, we transfected anti-human DAP1 hammerhead ribozymes into two colorectal tumour cell lines, HRT18 and HT115, and investigated the role of DAP1 through an in vitro cell function test. There was no significant difference in the growth rate between DAP1-knockdown cell lines and the corresponding controls. However, the in vitro cell-matrix adhesion assay and wound healing assay both indicated that DAP1-knockdown HRT18 and HT115 cancer cell lines presented more aggressive behaviour than the corresponding control cell lines. Faster wound 

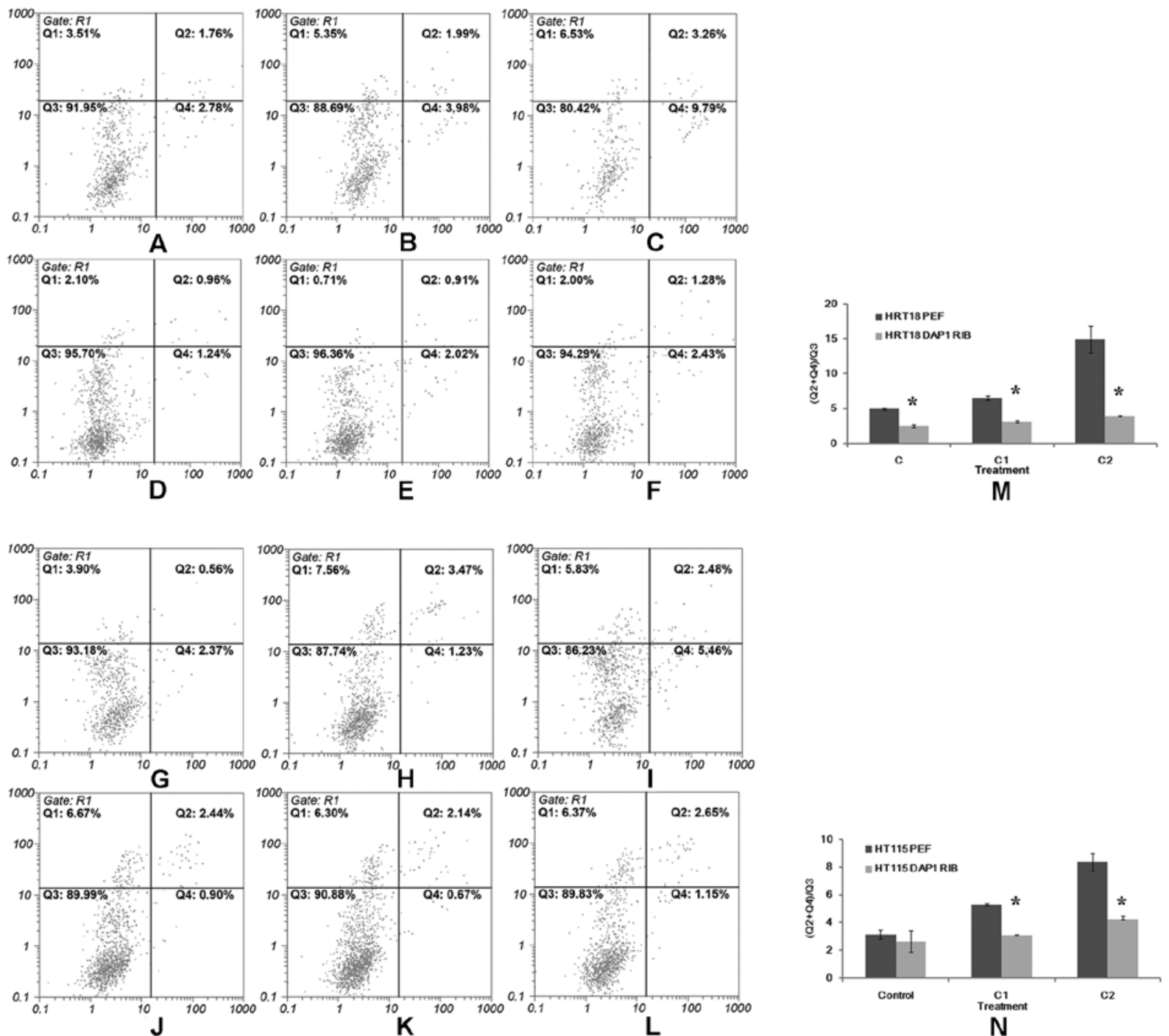

Figure 5. Flow cytometric analysis of apoptosis following treatment with a combination of 5-FU and oxaliplatin. (A-C) Flow cytometric analysis of apoptosis of the HRT18PEF cell line under a normal condition, treatment with combination of 5-FU and oxaliplatin at a lower and higher concentration. (D-F) Flow cytometric analysis of apoptosis of the HRT18DAP1RIB cell line under a normal condition, treatment with a combination of 5-FU and oxaliplatin at a lower and higher concentration. (G-I) Flow cytometric analysis of apoptosis of the HT115PEF cell line under a normal condition, treatment with combination of 5-FU and oxaliplatin at a lower and higher concentration. (J-L) Flow cytometric analysis of apoptosis of HT115DAP1RIB cell line under a normal condition, treatment with combination of 5-FU and oxaliplatin at a lower and higher concentration. (M) Proportion of HRT18PEF and HRT18DAP1RIB cells undergoing apoptosis under a normal condition and following treatment with a combination of 5-FU and oxaliplatin at a lower and higher concentration. (N) Proportion of HT115PEF and HRT115DAP1RIB cells undergoing apoptosis under a normal condition and following treatment with a combination of 5-FU and oxaliplatin at a lower and higher concentration. " $\mathrm{p}<0.01$. $\mathrm{C} 1$, lower concentration of $2 \times 10^{-6} \mathrm{M} ; \mathrm{C} 2$, higher concentration of $1 \times 10^{-5} \mathrm{M}$.

closure speed and stronger adherent ability were observed in the DAP1-RIB-transfected cell lines, which was consistent with the clinical cohort qPCR result that lower expression of DAP1 is correlated with tumour invasiveness. However only the HT115 DAP1RIB cell line exhibited invasive behaviour, which is only partially consistent with the clinical data indicating that patients with higher $\mathrm{T}$ stage or invasive tumours have a lower DAP1 expression level.

In the TKO strategy, IFN- $\gamma$ was initially utilized as a killing agent to induce cell apoptosis, and later various apoptosis-inducing stimuli such as TNF- $\alpha$ and FAS were all found to be able to be adopted as killing agents. Taken together, with the DAP1 expression patterns in colorectal cancer tissue and paired background normal tissue and the results of the cell function tests, it can be concluded that downregulation of DAP1 is part of the complicated development of tumour progression. Resistance to apoptotic stimuli by virtue of DAP1 downregulation may confer tumour cells a better chance to elude attacks from the immune system. This may be correlated with the results from the clinical cohort.

In the present study, we also investigated the growth rate of different cell lines exposed to 5-FU and oxaliplatin. The DAP1-knockdown cells exhibited higher tolerance to the genotoxic agents and higher growth rates, when compared with the control or wild-type cells. Thus, we can conclude that downregulation of DAP1 expression prevents colorectal tumour cell lines from undergoing apoptosis induced by chemotherapeutic agents and contributes to resistance to chemotherapy. When we consider the clinical cohort data, we found that, although chemotherapy information was not complete, a trend was observed that among the patients who received adjuvant chemotherapy or chemoradiotherapy, those 
with higher DAP1 expression had prolonged survival when compared with those with lower DAP1 expression. At present, DAP1 has only been reported to be involved in the extrinsic apoptosis pathway, but there are a number of studies indicating that DAP3 is a mitochondrial protein and is involved in the regulation of mitochondria morphology during the process of apoptosis (21-25). As a member of the DAP family, DAP1 may share many common molecular structures with DAP3 and may also be involved in the endogenous apoptosis pathway. However, the detailed mechanism of DAP1-related apoptosis remains to be elucidated.

In conclusion, DAP1 expression is downregulated in colorectal cancer and is correlated with advanced TNM/Dukes' stage and disease-free survival; knockdown of DAP1 expression enhanced the ability of HRT18 and HT115 cell lines to adhere and migrate. The resistance to chemotherapeutic agents may also be promoted by the downregulation of DAP1 expression. DAP1 may act as a tumour-suppressor gene in colorectal adenocarcinoma.

\section{Acknowledgements}

The authors wish to thank the Cancer Research Wales, and the Albert Hung Foundation for supporting the present study. Dr Y.J. is a recipient of the Cardiff University's China Medical Scholarship.

\section{References}

1. Inbal B, Shani G, Cohen O, Kissil JL and Kimchi A Death-associated protein kinase-related protein 1, a novel serine/threonine kinase involved in apoptosis. Mol Cell Biol 20: 1044-1054, 2000.

2. Cohen O, Inbal B, Kissil JL, et al: DAP-kinase participates in TNF- $\alpha-$ and Fas-induced apoptosis and its function requires the death domain. J Cell Biol 146: 141-148, 1999.

3. Inbal B, Cohen O, Polak-Charcon S, et al: DAP kinase links the control of apoptosis to metastasis. Nature 390: 180-184, 1997.

4. Lehmann U, Celikkaya G, Hasemeier B, Länger F and Kreipe H: Promoter hypermethylation of the death-associated protein kinase gene in breast cancer is associated with the invasive lobular subtype. Cancer Res 62: 6634-6638, 2002.

5. Green DR and Reed JC: Mitochondria and apoptosis. Science 281: $1309-1312,1998$

6. Korsmeyer SJ, Wei MC, Saito M, Weiler S, Oh KJ and Schlesinger PH: Pro-apoptotic cascade activates BID, which oligomerizes BAK or BAX into pores that result in the release of cytochrome $c$. Cell Death Differ 7: 1166-1173, 2000.

7. Kroemer G and Reed JC: Mitochondrial control of cell death. Nat Med 6: 513-519, 2000.
8. Martinou JC and Green DR: Breaking the mitochondrial barrier. Nat Rev Mol Cell Biol 2: 63-67, 2001.

9. Sugita $\mathrm{H}$, Iida $\mathrm{S}$, Inokuchi $\mathrm{M}$, et al: Methylation of BNIP3 and $D A P K$ indicates lower response to chemotherapy and poor prognosis in gastric cancer. Oncol Rep 25: 513-518, 2011.

10. Jiang WG, Watkins $\mathrm{G}$, Lane $\mathrm{J}$, et al: Prognostic value of rho GTPases and rho guanine nucleotide dissociation inhibitors in human breast cancers. Clin Cancer Res 9: 6432-6440, 2003.

11. Nazarenko IA, Bhatnagar SK and Hohman RJ: A closed tube format for amplification and detection of DNA based on energy transfer. Nucleic Acids Res 25: 2516-2521, 1997.

12. Zuker M: Mfold web server for nucleic acid folding and hybridization prediction. Nucleic Acids Res 31: 3406-3415, 2003.

13. Jiang WG, Hiscox S, Hallett MB, Scott C, Horrobin DF and Puntis MC: Inhibition of hepatocyte growth factor-induced motility and in vitro invasion of human colon cancer cells by gamma-linolenic acid. Br J Cancer 71: 744-752, 1995.

14. Jiang WG, Hiscox SE, Parr C, et al: Antagonistic effect of NK4, a novel hepatocyte growth factor variant, on in vitro angiogenesis of human vascular endothelial cells. Clin Cancer Res 5: 3695-3703, 1999.

15. Ye L, Kynaston $\mathrm{H}$ and Jiang WG: Bone morphogenetic protein-9 induces apoptosis in prostate cancer cells, the role of prostate apoptosis response-4. Mol Cancer Res 6: 1594-1606, 2008.

16. Kissil JL, Feinstein E, Cohen O, et al: DAP-kinase loss of expression in various carcinoma and B-cell lymphoma cell lines: possible implications for role as tumor suppressor gene. Oncogene 15: 403-407, 1997.

17. Esteller M, Sanchez-Cespedes M, Rosell R, Sidransky D, Baylin SB and Herman JG: Detection of aberrant promoter hypermethylation of tumor suppressor genes in serum DNA from non-small cell lung cancer patients. Cancer Res 59: 67-70, 1999.

18. Sanchez-Cespedes M, Esteller M, Wu L, et al: Gene promoter hypermethylation in tumors and serum of head and neck cancer patients. Cancer Res 60: 892-895, 2000.

19. Sasaki H, Ide N, Yukiue H, et al: Arg and DAP3 expression was correlated with human thymoma stage. Clin Exp Metastasis 21: 507-513, 2004

20. Jacques C, Fontaine JF, Franc B, et al: Death-associated protein 3 is overexpressed in human thyroid oncocytic tumours. Br J Cancer 101: 132-138, 2009.

21. Suzuki T, Terasaki M, Takemoto-Hori C, et al: Proteomic analysis of the mammalian mitochondrial ribosome. Identification of protein components in the $28 \mathrm{~S}$ small subunit. J Biol Chem 276: 33181-33195, 2001.

22. Cavdar Koc E, Burkhart W, Blackburn K, Moseley A and Spremulli LL: The small subunit of the mammalian mitochondrial ribosome. Identification of the full complement of ribosomal proteins present. J Biol Chem 276: 19363-19374, 2001.

23. Zhang $Z$ and Gerstein $M$ : Identification and characterization of over 100 mitochondrial ribosomal protein pseudogenes in the human genome. Genomics 81: 468-480, 2003.

24. Mukamel Z and Kimchi A: Death-associated protein 3 localizes to the mitochondria and is involved in the process of mitochondrial fragmentation during cell death. J Biol Chem 279: 36732-36738, 2004.

25. Cavdar Koc E, Ranasinghe A, Burkhart W, et al: A new face on apoptosis: death-associated protein 3 and PDCD9 are mitochondrial ribosomal proteins. FEBS Lett 492: 166-170, 2001. 